\title{
An Elastica-driven Digital Curve Evolution Model for Image Segmentation
}

\author{
Daniel Antunes · Jacques-Olivier \\ Lachaud • Hugues Talbot
}

the date of receipt and acceptance should be inserted later

\begin{abstract}
Geometric priors have been shown to be useful in image segmentation to regularize results. For example, the classical Mumford-Shah functional uses region perimeter as prior. This has inspired much research in the last few decades, with classical approaches like the Rudin-Osher-Fatemi and most graph-cut formulations, which all use a weighted or binary perimeter prior. It has been observed that this prior is not suitable in many applications, for example for segmenting thin objects or some textures, which may have high perimeter/surface ratio. Mumford observed that an interesting prior for natural objects is the Euler Elastical model, which involves the squared curvature. In other areas of science, researchers have noticed that some physical binarization processes, like emulsion unmixing can be well-approximated by curvature-related flow like the Willmore flow. However, curvature-related flows are not easy to compute because curvature is difficult to estimate accurately, and the underlying optimisation processes are not convex. In this article, we propose to formulate a digital flow that approximates an Elastica-related flow using a multigrid-convergent curvature estimator, within a discrete variational framework. We also present an application of this model as a post-processing step to a segmentation framework.
\end{abstract}

Keywords Multigrid convergence · Digital estimator · Curvature · Shape Optimization · Image Segmentation.

This work has been partially funded by CoMeDiC ANR-15-CE40-0006 research grant.

Daniel Antunes

Univ. Grenoble Alpes, Univ. Savoie Mont Blanc, CNRS, LAMA, 73000 Chambéry, France E-mail: daniel.martins-antunes@univ-smb.fr

Jacques-Olivier Lachaud

Univ. Grenoble Alpes, Univ. Savoie Mont Blanc, CNRS, LAMA, 73000 Chambéry, France E-mail: jacques-olivier.lachaud@univ-smb.fr

Hugues Talbot

CentraleSupelec, Inria, Université Paris-Saclay, 9 rue Joliot-Curie, F-91190 Gif-sur-Yvette E-mail: hugues.talbot@centralesupelec.fr 


\section{Introduction}

Geometric quantities are particularly useful as regularizers in low-level image analysis, especially when little prior information is known about the shape of interest. Length penalization is a well-behaved, general purpose regularizer and many models in the literature make use of it, from active contours [8] to level-set formulations [26,27]. Discrete graph-based variational models have been particularly succesful to incorporate length penalization as a penalizer, while keeping the ability to extract a global optimum [5,2].

However length regularization shows limitations when segmenting small or thin and elongated objects, as it tends to shrink solutions or yields disconnected solutions. Such drawbacks can be problematic in image segmentation, image restoration or image inpainting. It is thus rather natural to consider curvature (especially squared curvature) as a potential regularizer. Energies involving both length and squared curvature are often called the Elastica model (that dates back to Euler). The Willmore energy is its $n$-dimensional extension. These were brought to attention in computer vision by Mumford [31]. A similar concept is also present in the second-order regularizer of the original snake model [20].Indeed, an explicit curvature term was often employed in early level-set methods, like $[26,27,3]$ for segmentation or inpainting. However, Casselles et al. in [8] observed that these terms as employed were not geometric, in the sense that they depend on the discretization parameters.

\subsection{Existing works}

The use of curvature for surface regularization in the case of the Willmore energy was studied in [4]. Although results look promising, authors used an inexact linearized version of the square curvature at every step.

One of the first successful uses of geometric, squared curvature in image processing is the inpainting algorithm described in [30], where authors evaluate the absolute curvature along the level lines of a simply-connected image to reconstruct its occluded parts. The non-intersection property of level lines induces the construction of an efficient dynamic programming algorithm. The curvature energy is nevertheless only coarsely approximated. We may quote [9] as another geometric inpainting method involving Elastica, which is optimized with level-sets.

In image segmentation, Zehiry and Grady have shown that injecting curvature as a regularizer can help recover thin and elongated objects [15]. Similarly Schoenemann et al. [38] have considered the Elastica energy in their ratiobased image segmentation approach. In [39], Schoenemann et al. extend ratiocut approaches to segmentation with a curvature term. Their global optimization framework shows the considerable advantages of using such regularizer in common binary image segmentation tasks. However the time complexity of their algorithm is prohibitive, even if curvature is again coarsely approximated. In a more recent work, Lim et al. propose an edge-weighted Willmore-like flow 
to segment vertebra [25] using a level-set formulation. They observe improved results compared to [8] and other perimeter-based priors, but observe that a shape prior and a close-enough initialization must also be provided to achieve good results.

In fact, it is still a very challenging task to efficiently handle curvature in the context of image segmentation. State-of-art methods are in practice difficult to optimize and do not scale easily $[15,38,40,32]$. In order to achieve reasonable running times, such approaches make use of coarse curvature estimations for which the approximation error is often unknown. Improving the quality of the curvature estimator has an important impact on the accuracy of the results, but is computationally too costly in these methods.

Segmentation methods using Elastica or Willmore energies can be formulated via the computation of the corresponding flow, i.e. following its gradient. Well-founded level-set methods involving Willmore energy [14] have been used in medical image segmentation [25]. Another level-set formulation similar to the Chan-Vese model was proposed in [42]. Willmore flow can also be carried out by phase-field models (see [7]) but they are less suited to image segmentation since interfaces are blurred in such models. Threshold dynamics can also be considered for such energies [18] and has been proposed for image desocclusion [17]. However, flow-based methods will always deliver a local minimum, which heavily depend on initialization for good results. A similar approach is to formulate the Elastica as a regularization in a variational framework and use operator splitting to optimize it. Tai et al. [41] and more recently Deng et al. [13] have proposed this approach, which is well-suited to inverse problem solving. The problem remains non-convex and the nature of the converged iterates are not studied, i.e. how close they are to a global optimum.

In contrast, researchers have sought convex relaxation of curvature-related formulation. In [19], Goldluecke and Cremers introduce the Total Curvature, based on the Menger-Melnikov curvature of a measure. The formulation is non-convex but can be relaxed into a well-correlated but non-tight convex one. The relaxed formulation can be used to solve inverse problems including segmentation. However computing a solution is still expensive, requiring signifiant parallelisation efforts with a GPU to achieve acceptable computing times. In [6], Bredies et al. study a convex approximation of the Elastica energy, using a lifting scheme. Thanks to the lifting, it is not restricted to binary sets. It shows promising results and can be used for image restoration in addition to inpainting and segmentation. The scheme is however neither tight nor exact, and required 4 dimensions for a 2D approximation. In addition, it does not carry over to $n D$. It also remains computationally expensive.

Discrete approaches have been used to tackle the Elastica difficulties. As noted before, [38] have linearized a mesh-based approach, which was expanded upon by [40], while [15] have used a binary solver for a non-submodular approach on a regular square grid of pixels, which was expanded in [16]. In [32] , a specific solver is developed to yield a better solution to an extension of the formulation in [15]. 


\subsection{Motivation}

In spite of the multiplicity of existing approaches, it seems none is currently completely satisfactory. While the sought solution is expected to be very smooth based on geometrical expectations, the mathematical properties of the related operators make this smoothness elusive. Non-convexity and the presence of high-order terms make Elastica difficult to optimize and to approximate, let alone compute efficiently. The computed solutions in the literature are rarely evaluated, and the complexity of the proposed solution is often high.

Consequently, we are interested in studying purely discrete variational segmentation models involving an Elastica energy. Recently, new estimators of curvature along digital contours have been proposed $[36,11,37]$ with the desirable multigrid convergence property. This motivates us to propose models in which they can be used successfully.

In this paper, we propose to investigate the use of the digital integral invariant curvature estimator [11] in a discrete variational segmentation framework. More precisely, we show how to incorporate it in a digital flow minimizing its squared curvature. This model is expressible as a discrete combinatorial optimization model, which we solve using a classical variant of QPBO algorithm [35]. Our solution does not compute a global solution to the whole domain but a global solution to a band surrounding a given shape. Our approach thus leads to a digital flow similar to continuous Elastica, as shown by our experiments. We present an application of this model as a post-processing step in a segmentation framework, and demonstrate how it can improve standard results. This paper is an extended version of a previous work that appeared in [1]. We have included a deeper discussion on our optimization method and we present a more thorough comparison of our segmentation method with two other related works. Moreover, we have included a new local combinatorial optimization approach for elastica minimization, which show that our discrete model is well-founded. Finally, the code produced in this paper is freely available on github. ${ }^{1}$

\subsection{Outline}

Section 2 reviews the concept of multigrid convergence and highlights its importance for the definition of digital estimators. We describe two convergent estimators used in this paper, one that approaches the length of elementary discrete contour elements, and the other that approaches the curvature of a discrete contour. They are used in the optimization model and in the definition of the digital elastica. Section 3 describes a local combinatorial optimization model suitable for several curvature estimators, that presents interesting results but is too costly in practice. Section 4 describes the proposed Elasticadriven curvature evolution model along with several illustrations of digital flows. Section 5 explains how to use this evolution model as a post-processing

\footnotetext{
1 https://www.github.com/danoan/BTools
} 
step in an image segmentation framework and compares it to two related approaches. We conclude and draw some perspectives to this work in Section 6 .

\section{Multigrid convergent estimators}

Our objective is to delineate shapes within digital images with some priors related to continuous geometry. The goal of this section is to introduce the concept of multigrid convergence and its potential when analyzing digital images with variational models involving geometric quantities, with the constraint that only digitized shapes are observed.

A digital shape is the result of some quantization process over an object $X$ lying in some continuous space of dimension 2 (here). For example, the Gauss digitization of a continuous subset $X$ of the Euclidean plane $\mathbb{R}^{2}$ with grid step $h>0$ is defined as

$$
D_{h}(X)=X \cap(h \mathbb{Z})^{2}
$$

Given a shape $X$ and its digitization $D_{h}(X)$, a digital estimator $\hat{u}$ for some geometric quantity $u$ is intended to compute $u(X)$ by using only the digitization. This problem is not well-posed, as the same digital object could be the digitization of infinitely many objects very different from $X$. Therefore, a characterization of what constitutes a good estimator is necessary.

Let $u$ be some geometric quantity of $X$ (e.g. tangent, curvature). We wish to devise a digital estimator $\hat{u}$ for $u$. It is reasonable to state that $\hat{u}$ is a good estimator if $\hat{u}\left(D_{h}(X)\right)$ converges to $u(X)$ as we refine our grid. For example, counting pixels is a convergent estimator for area (with a rescale of $h^{2}$ ); but counting boundary pixels (with a rescale of $h$ ) is not a convergent estimator for perimeter. Multigrid convergence is the mathematical tool that makes this definition precise. Given any subset $Z$ of $(h \mathbb{Z})^{2}$, we can represent it as a union of axis-aligned squares with edge length $h$ centered on the point of $Z$. The topological boundary of this union of cubes is called $h$-frontier of $Z$. When $Z=D_{h}(X)$, we call it $h$-boundary of $X$ and denote it by $\partial_{h} X$.

Definition 1 (Multigrid convergence for local geometric quantites) A local discrete geometric estimator $\hat{u}$ of some geometric quantity $u$ is (uniformly) multigrid convergent for the family $\mathbb{X}$ if and only if, for any $X \in \mathbb{X}$, there exists a grid step $h_{X}>0$ such that the estimate $\hat{u}\left(D_{h}(X), \hat{x}, h\right)$ is defined for all $\hat{x} \in \partial_{h} X$ with $0<h<h_{X}$, and for any $x \in \partial X$,

$$
\forall \hat{x} \in \partial_{h} X \text { with }\|\hat{x}-x\|_{\infty} \leq h,\left\|\hat{u}\left(D_{h}(X), \hat{x}, h\right)-u(X, x)\right\| \leq \tau_{X}(h),
$$

where $\tau_{X}: \mathbb{R}^{+} \backslash\{0\} \rightarrow \mathbb{R}^{+}$has null limit at 0 . This function defines the speed of convergence of $\hat{u}$ towards $u$ for $X$.

For a global geometric quantity (e.g. perimeter, area, volume), the definition remains the same, except that the mapping between $\partial X$ and $\partial_{h} X$ is no longer necessary. 
Multigrid convergent estimators provide a quality guarantee and should be preferred over non-multigrid convergent ones. In the next section, we describe two estimators that are important for our purpose.

\subsection{Tangent and Perimeter Estimators}

The literature presents several perimeter estimators that are multigrid convergent (see $[10,12]$ for a review), but in order to define the digital Elastica we need a local estimation of length and we wish that integration over these local length elements gives a multigrid convergent estimator for the perimeter.

Definition 2 (Elementary Length) Let a digital curve $C$ be represented as a sequence of grid vertices in a grid cell representation of digital objects (in a grid with step $h$ ). Further, let $\hat{\mathbf{v}}$ be a multigrid convergent estimator for the unit tangent vector. The elementary length $\hat{s}(\mathbf{e})$ at some oriented grid edge $\mathbf{e} \in C$ is defined as

$$
\hat{s}(\mathbf{e})=h \hat{\mathbf{v}}(\mathbf{e}) \cdot \mathbf{e} .
$$

The integration of the elementary length along the digital curve is a multigrid convergent estimator for perimeter if one uses the $\lambda$-MST [24] tangent estimator (see [23]).

\subsection{Integral Invariant Curvature Estimator}

Generally speaking, an invariant is a function whose value is unaffected by the action of some group on the elements of the domain. Perimeter and curvature are examples of invariants for shapes on $\mathbb{R}^{2}$ with respect to the euclidean group of rigid transformations. Definition of integral area invariant and its link with curvature is proven in [28].

Definition 3 (Integral area invariant) Let $X \subset \mathbb{R}^{2}$ and $B_{r}(p)$ the ball of radius $r$ centered at point $p$. Further, let $\mathbb{1}_{X}(\cdot)$ be the characteristic function of $X$. The integral area invariant $\sigma_{X, r}(\cdot)$ is defined as

$$
\forall p \in \partial X, \quad \sigma_{X, r}(p)=\int_{B_{r}(p)} \mathbb{1}_{X}(x) d x .
$$

The value $\sigma_{X, r}(p)$ is the area of the intersection of the ball $B_{r}(p)$ with shape $X$. By approaching the shape at point $p \in X$, one can rewrite the intersection area $\sigma_{X, r}(p)$ in the form of the Taylor expansion [33]:

$$
\sigma_{X, r}(p)=\frac{\pi}{2} r^{2}-\frac{\kappa(X, p)}{3} r^{3}+O\left(r^{4}\right)
$$

where $\kappa(X, p)$ is the curvature of $X$ at point $p$. By isolating $\kappa$ we can define a curvature estimator 


$$
\tilde{\kappa}(p):=\frac{3}{r^{3}}\left(\frac{\pi r^{2}}{2}-\sigma_{X, r}(p)\right),
$$

Such an approximation is convenient as one can simply devise a multigrid convergent estimator for the area.

Definition 4 Given a digital shape $D \subset(h \mathbb{Z})^{2}$, the area estimatir $\widehat{\operatorname{Area}}(D, h)$ is defined as

$$
\widehat{\operatorname{Area}}(D, h):=h^{2} \operatorname{Card}(D)
$$

It is well known that this area estimator is multigrid convergent (e.g. see [21]). In [11], the authors combine the approximation(1) and digital estimator (2) to define a multigrid convergent estimator for the curvature.

Definition 5 (Integral Invariant Curvature Estimator) Let $D \subset(h \mathbb{Z})^{2}$ a digital shape. The integral invariant curvature estimator is defined as

$$
\hat{\kappa}_{r}(D, x, h):=\frac{3}{r^{3}}\left(\frac{\pi r^{2}}{2}-\widehat{\operatorname{Area}}\left(B_{r}(x) \cap D, h\right)\right) .
$$

This estimator is multigrid convergent with speed $O\left(h^{\frac{1}{3}}\right)$ for radii chosen as $r=\Theta\left(h^{\frac{1}{3}}\right)$. This estimator is also robust to noise and can be extended to estimate the mean curvature of three dimensional shapes.

\subsection{Elastica energy estimator}

In the remaining of this article, we are considering in the minimization of the Elastica energy. Given a Euclidean shape $X$ with smooth enough boundary, the Elastica is defined as

$$
E(X)=\int_{\partial X}\left(\alpha+\beta \kappa^{2}\right) d s, \quad \text { for } \alpha \geq 0, \beta \geq 0 .
$$

The digital version of Elastica energy approaching the continuous Elastica energy is defined as follows (it uses multigrid convergent estimators):

$$
\hat{E}\left(D_{h}(X)\right)=\sum_{\mathbf{e} \in \partial D_{h}(X)} \hat{s}(\mathbf{e})\left(\alpha+\beta \hat{\kappa}_{r}^{2}\left(D_{h}(X), \dot{\mathbf{e}}, h\right)\right),
$$

where $\dot{\mathbf{e}}$ denotes the center of the edge e. In the expression above, we will substitute an arbitrary subset $Z$ of $\mathbb{Z}^{2}$ to $D_{h}(X)$ since the continuous shape $X$ is unknown. In the following we omit the grid step $h$ to simplify expressions (or, putting it differently, we assume that the shape of interest is rescaled by $1 / h$ and we set $h=1)$. 


\section{Local combinatorial optimization}

The objectives of this section is to show both the potential of minimizing a purely digital Elastica energy but also to underline the difficulties of using it in a global combinatorial optimization framework.

Given a digital shape $S^{(0)}$ we describe a process that generates a sequence $S^{(i)}$ of shapes with non-increasing Elastica energy. The idea is to define a neighborhood of shapes $\mathcal{N}^{(i)}$ to the shape $S^{(i)}$ and choose the element of $\mathcal{N}^{(i)}$ with lowest energy. The process is suited for the integral invariant estimator but also for other curvature estimators, for example, MDCA [36]. As a matter of fact, our experiments have shown that either estimators induce similar results.

Let $S$ be a 2-dimensional digital shape. We adopt the cellular-grid model to represent $S$, i.e., pixels and its lower dimensional counterparts, linels and pointels, are part of $S$. In particular, we denote by $\partial S$ the topological boundary of $S$, i.e., the connected sequence of linels such that for each linel we have one of its incident pixels in $S$ and the other not in $S$.

Let $d_{S}: \Omega \rightarrow \mathcal{R}$ be the Euclidean distance transformation with respect to shape $S$. The value $d_{S}(x)$ gives the Euclidean distance between $x$ and the closest pixel in $S$.

Definition 6 (m-Ring Set) Given a digital shape $S \in \Omega$, its distance transformation $d_{S}$ and natural numbers $m>0$, the $m$-ring set of $S$ is defined as

$$
\begin{aligned}
R_{m}(S) & :=R_{m}^{-}(S) \cup R_{m}^{+}(S) \\
& :=\left\{x \in \Omega \mid m-1<d_{S}(x) \leq m\right\} \cup\left\{x \in \Omega \mid m-1<d_{\bar{S}}(x) \leq m\right\}
\end{aligned}
$$

Consider the following set of neighbor candidates to $S$ :

$$
\mathcal{U}(S)=\left\{D \mid D \subset R_{1}(S) \cup S \text { and } D \text { is connected }\right\}
$$

Such set can be extremely large and its complete exhaustion is prohibitively expensive. Instead, we explore a subset of it with the help of $n$-glued curves.

An oriented closed curve $C$ is a closed connected sequence of linels with a well-defined interior. A segment of $C$ is a connected subsequence $c \in C$ of its linels.

Definition 7 (Glued Curve) Given closed curves $C_{1}, C_{2}$ agreeing with some orientation $q$, a glued curve is a closed curve $\left(c_{1}, \ell_{1}, c_{2}, \ell_{2}\right)$ with orientation $q$ and $c_{1} \in C_{1}, c_{2} \in C_{2}$. The linels $\ell_{1}, \ell_{2}$ are called junction linels.

Definition 8 ( $n$-Glued Curve Set) Given closed curves $C_{1}, C_{2}$ with same orientation, its set of $n$-glued curves is defined as

$$
\mathcal{G}_{n}\left(C_{1}, C_{2}\right)=\left\{\left(c_{1}, \ell_{1}, c_{2}, \ell_{2}\right)|| c_{2} \mid=n\right\}
$$




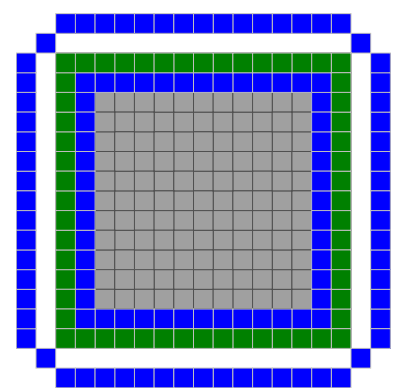

(a)

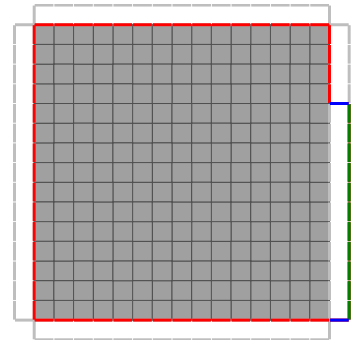

(b)

Fig. 1: The blue pixels in figure (a) illustrates a 2-ring set, while the green pixels its inner-pixel boundary. In figure (b) we highlight an element of set $\mathcal{G}_{11}\left(C_{1}, C_{2}\right)$. The curve segments of $C_{1}$ and $C_{2}$ are colored in red and green, while the junction linels in blue.

Let $S_{O}=\left(S \cup R_{1}^{+}(S)\right)$ and $S_{I}=\left(S \backslash R_{1}^{-}(S)\right)$, the neighborhood set to shape $S$ is defined as

$$
\mathcal{N}(S, N)=\bigcup_{1 \leq n \leq N} \operatorname{int}\left(\mathcal{G}_{n}\left(\partial S_{O}, \partial S\right)\right) \cup \operatorname{int}\left(\mathcal{G}_{n}\left(\partial S, \partial S_{I}\right)\right),
$$

where $\operatorname{int}(C)$ is the interior of the shape bounded by the close oriented curve $C$. Algorithm 11 describes the local combinatorial process and Figure 2 presents the digital curve evolution when executing this algorithm for two different shapes with $N=20$.

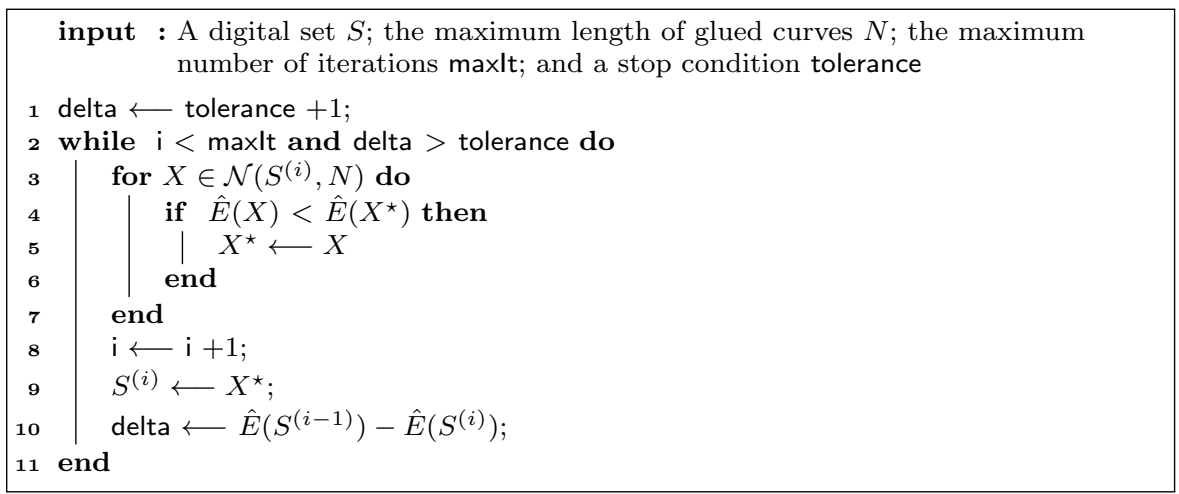

Algorithm 1: Local combinatorial optimization for elastica minimization.

The running time of algorithm 11 is summarized in table 1 . All the experiments in this paper were executed on a five-core 3.4Ghz CPU and the number of pixels in the triangle, square and flower shapes are respectively 


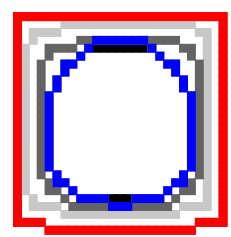

(a) $\mathrm{h}=1.0$

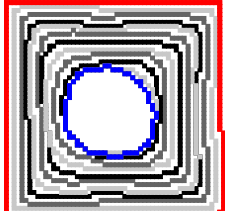

(b) $\mathrm{h}=0.5$

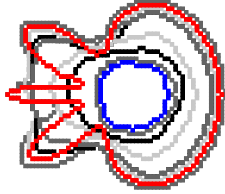

(c) $\mathrm{h}=1.0$

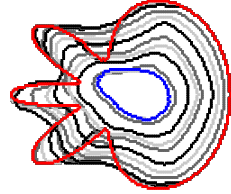

(d) $\mathrm{h}=0.5$

Fig. 2: Local combinatorial optimization process results for the square and flower shapes. Shapes displayed at every 2 iterations for the square and 7 iterations for the flower.

$841,1867,521$ for grid step $h=1.0$. Although its use in practical applications is limited, we demonstrate that digital estimators are effective in their measurements and the flows evolve as expected. We observe that it is a complete digital approach, and we do not suffer from discretization and rounding problems, a common issue in continuous models. Furthermore we have checked that this approach works indifferently with Integral Invariant curvature estimator and Maximal Digital Circular Arc curvature estimator. So the convergence of the digital curvature estimator seems to be the cornerstone to get a digital curve behaving like a continuous Elastica. In the next section, we explore a more efficient approach.

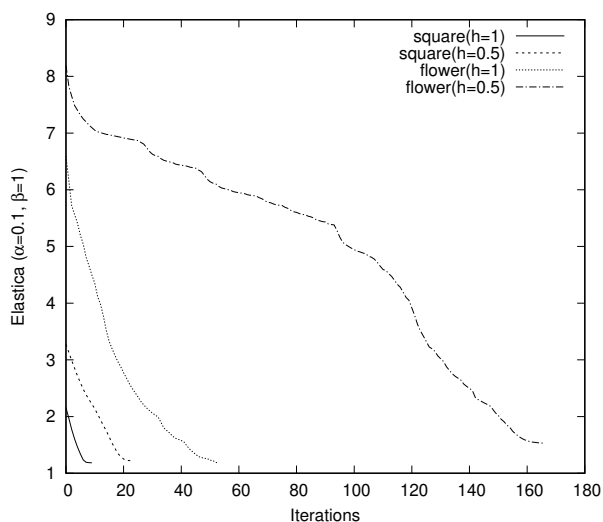

Fig. 3: Elastica computation along iterations of algorithm 11

\begin{tabular}{|c|c|c|}
\hline & $h=1.0$ & $h=0.5$ \\
\hline Square & $47 \mathrm{~s}(5 \mathrm{~s} / \mathrm{it})$ & $260 \mathrm{~s}(12 \mathrm{~s} / \mathrm{it})$ \\
\hline Flower & $1235 \mathrm{~s}(23 \mathrm{~s} / \mathrm{it})$ & $8516 \mathrm{~s}(52 \mathrm{~s} / \mathrm{it})$ \\
\hline
\end{tabular}

Table 1: Running times for the local combinatorial optimization algorithm with $k=1, N=20$. Four threads were used.

\section{Digital Curvature Flow}

Given a digital shape $S \in \Omega$, the idea is to evolve a flow $S^{(i)}$ where $S^{(i+1)}$ is derived from the minimization of the sum of the squared curvature at $S^{(i)}$. In 
order to guarantee connectivity, we limit the optimization region to a subset of $\Omega$, namely the inner pixel boundary of $S^{(i)}$. We also use the notation $|S|$ to denote the cardinal of a digital set $S$.

4.1 Discrete variational model for discrete curve evolution

We start by definition the optimization variables.

Definition 9 (Inner pixel boundary) Given a digital shape $S$ embedded in a domain $\Omega$, we define its inner pixel boundary set $O(S)$ as

$$
O(S)=\left\{x|x \in S,| \mathcal{N}_{4}(x) \cap S \mid<4\right\},
$$

where $\mathcal{N}_{4}(x)$ denotes the 4 -adjacent neighbor set of $x$ (without $x$ ).

In other words, each flow iteration decides which pixels in the inner boundary are to be removed and which are to be kept. To simplify notation, we denote the optimization region of $S^{(i)}$ as $O^{(i)}$, and the foreground set as $F^{(i)}=S^{(i)} \backslash O^{(i)}$. We recall the definition of our digital curvature estimator :

$$
\hat{\kappa}^{2}(y)=c_{1}\left(c_{2}-\left|B_{r}(y) \cap S\right|\right)^{2},
$$

where $c_{1}=3 / r^{6}$ and $c_{2}=\pi r^{2} / 2$.

We define $O_{r}^{(i)}(y):=O^{(i)} \cap B_{r}(y)$ and an analogous definition holds for $F_{r}^{(i)}(y)$. Expanding (5), we get

$$
\begin{aligned}
\hat{\kappa}^{2}(y) & =c_{1}\left(c_{2}-\sum_{x_{j} \in B_{r}(y)}\left(x_{j}+\left|F_{r}^{(i)}(y)\right|\right)\right)^{2} \\
& =c_{1} \cdot\left(C+2\left(\left|F_{r}^{(i)}(y)\right|-c_{2}\right) \sum_{x_{j} \in O_{r}^{(i)}(y)} x_{j}+\sum_{x_{j} \in O_{r}^{(i)}(y)} x_{j}^{2}+\sum_{\substack{x_{j}, x_{k} \in O_{r}^{(i)}(y) \\
j<k}} 2 x_{j} x_{k}\right),
\end{aligned}
$$

where $C=c_{2}^{2}-2 c_{2} \cdot\left|F_{r}^{(i)}(y)\right|+\left|F_{r}^{(i)}(y)\right|^{2}$ is a constant. By ignoring constants and multiplication factors and using the binary character of the variables, we define the following family of energies for given parameters $\alpha, \beta, \gamma \geq 0$.

$$
\begin{aligned}
E_{m}(X, S)= & \sum_{x \in X} \alpha s(x) \\
& +\sum_{y \in R_{m}(S)} \beta\left(\left(1 / 2+\left|F_{r}^{(i)}(y)\right|-c_{2}\right) \cdot \sum_{x_{j} \in O_{r}^{(i)}(y)} x_{j}+\sum_{\substack{x_{j}, x_{k} \in O_{r}^{(i)}(y) \\
j<k}} x_{j} x_{k}\right) \\
& +\sum_{x \in X} \gamma g(S, x),
\end{aligned}
$$

where $g(S, x)$ denotes a similarity term as distance to initial shape $S$ or data fidelity term and $s(x)$ denotes a length penalization term. Each choice of $m$ 
generates a different flow, which is generaly described in the Digital Curve Evolution (DCE) algorithm 15.

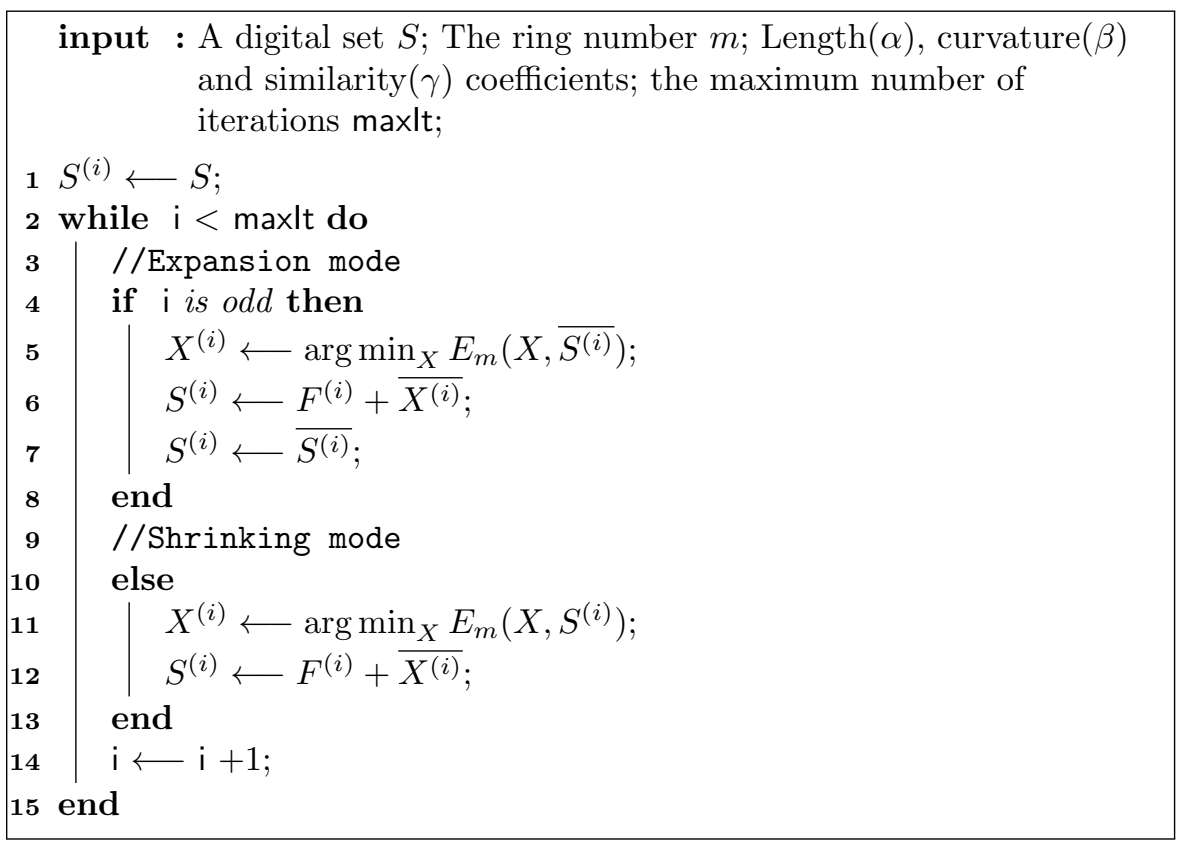

Algorithm 2: Digital curvature evolution algorithm (DCE).

Recall that the integral invariant estimator approaches curvature by computing the difference between half of the area of a chosen ball and the area of the intersection of this ball with the shape. In particular, regions of positive curvature have fewer pixels in their intersection set than on its complement w.r.t the estimation ball. This implies that variables in such regions are labeled with 1 , as the unbalance grows otherwise. We attenuate curvature if we shift the center of the estimation ball towards the interior of the shape, which means removing the 1-labeled pixels. That is why we take the complement of the optimization solution.

The same reasoning applies for non-convex parts. Indeed, concave regions are convex in the shape complement. In the expansion mode we apply the same reasoning on the image complement, and by doing this we are able to handle concavities. It is called expansion mode because the optimization region, in this case, is the outer pixel boundary of the original shape. Table 2 sums up these arguments.

Length and similarity terms should be properly defined in order to comply with the complement step of the DCE algorithm. The length penalization is defined as

$$
s(x)=\sum_{x_{j} \in \mathcal{N}_{4}(x)} t\left(x_{j}\right), \quad \text { where } t\left(x_{j}\right)= \begin{cases}\left(x-x_{j}\right)^{2}, & \text { if } x_{j} \in O_{r}^{(i)} \\ (x-0), & \text { if } x_{j} \in F_{r}^{(i)} \\ (x-1), & \text { otherwise }\end{cases}
$$




\begin{tabular}{|c|c|c|c|}
\hline shrink mode & $\kappa \gg 0$ & $\kappa \geq 0$ & $\kappa<0$ \\
\hline$X$ & $x_{k}=1$ & $x_{k} \in\{0,1\}$ & $x_{k}=0$ \\
\hline$S^{(i+1)} \leftarrow S^{(i)} \backslash X$ & eroded & prob. eroded & unchanged \\
\hline \hline expansion mode & $\bar{\kappa} \gg 0$ & $\bar{\kappa} \geq 0$ & $\bar{\kappa}<0$ \\
\hline$X$ & $\bar{x}_{k}=1$ & $\bar{x}_{k} \in\{0,1\}$ & $\bar{x}_{k}=0$ \\
\hline$S^{(i+1)} \leftarrow \overline{S^{(i)} \backslash \bar{X}}$ & dilated & prob. dilated & unchanged \\
\hline
\end{tabular}

Table 2: Since the curvature is negated when reversing the curve (i.e. $\bar{\kappa}=-\kappa$ ), this process can only shrink convex parts in shrink mode and expand concave parts in expansion mode.

We do not use similarity terms in this section, thus we postpone the definition of such terms until later in this article, with the description of how DCE can be used in an image segmentation framework.

In figure 4 we see some results for the DCE algorithm with $m=1$. We observe a global evolution towards rounder shapes, but with several artifacts. We minimize the effects of a jaggy boundary by setting $\alpha>0$. Nonetheless, a higher estimation ball radius creates unstable shapes. In fact, the estimator is very sensitive in regions of low squared curvature, and it is precisely in those regions that spurious pixels are created.

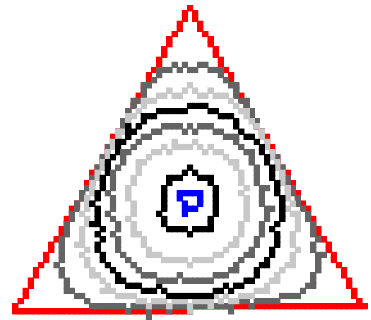

(a) $r=3, \alpha=0$

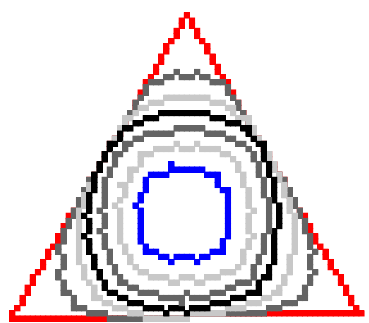

(b) $r=3, \alpha=0.15$

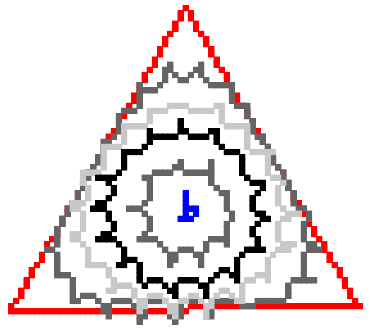

(c) $r=5, \alpha=0.15$

Fig. 4: The algorithm is very sensitive to the little variations of the estimator, which are particularly important in regions of low squared curvature. Artifacts are somewhat reduced with a length penalization but increases if we use a higher ball radius.

\subsection{A more stable model.}

In the previous section we noticed that the algorithm produces shapes with many artifacts due to the small uncertainties of the estimator along regions of low squared curvature. We argue that, evaluating the estimation ball along outer ring sets we avoid those sensitive areas by focusing the optimization process only on regions with highest squared curvature value. 


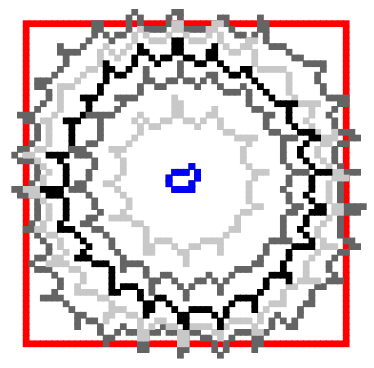

(a) $r=5, m=1$

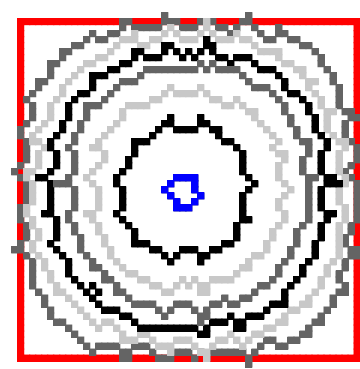

(b) $r=5, m=2$

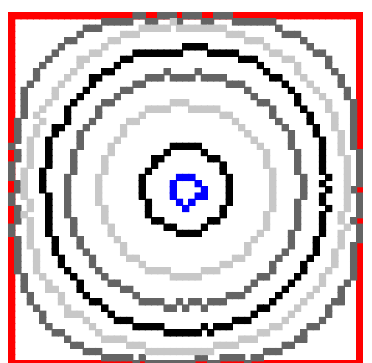

(c) $r=5, m=3$

Fig. 5: By positioning the estimation ball on farther rings, we minimize artifacts creation.

In our experiments, the best results are obtained by executing DCE algorithm with $m$ equal to $r$, where $r$ is the estimation ball radius (see figure 5 ). We observe that Elastica may increase after some iterations if chosen radius is too large, as in the case of the triangle in figure 6 in which the flow converges to a single point. We conjecture that an appropriated value for the radius should be given by the shape reach. The produced flow has no difficulties in handling changes on topology, and it presents different speeds for regions with low and high curvature values, as illustrated in figure 7 .

\subsection{Optimization method}

Let $f$ be a function of $n$ binary variables with unary and pairwise terms, i.e.

$$
f\left(y_{1}, \cdots, y_{n}\right)=\sum_{j} f_{j}\left(y_{j}\right)+\sum_{j<k} f_{j, k}\left(y_{j}, y_{k}\right) .
$$

Function $f$ is submodular if and only if the following inequality holds for each pairwise term $f_{j, k}[22]$ :

$$
f_{j, k}(0,0)+f_{j, k}(1,1) \leq f_{j, k}(0,1)+f_{j, k}(1,0) .
$$

Energy $E_{m}$ is non-submodular and optimizing it is a difficult problem, which constrains us to use heuristics and approximation algorithms. The QPBO method [35] transforms the original problem in a max-flow/min-cut formulation and yields a full optimal labeling for submodular energies. For nonsubmodular energies the method is guaranteed to return a partial labeling with the property that the set of labeled variables is part of an optimal solution. That property is called partial optimality.

In practice, QPBO can leave many pixels unlabeled. There exist two extensions to QPBO that alleviate this limitation: QPBOI (improve) and QPBOP 

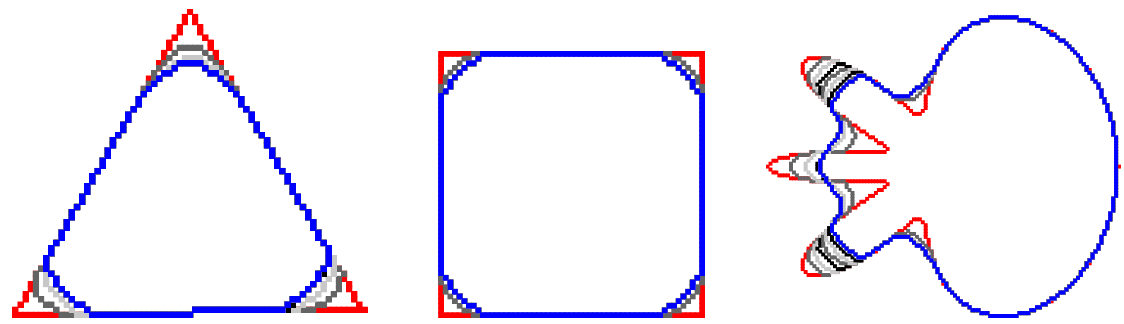

(a) $r=3, h=0.5$
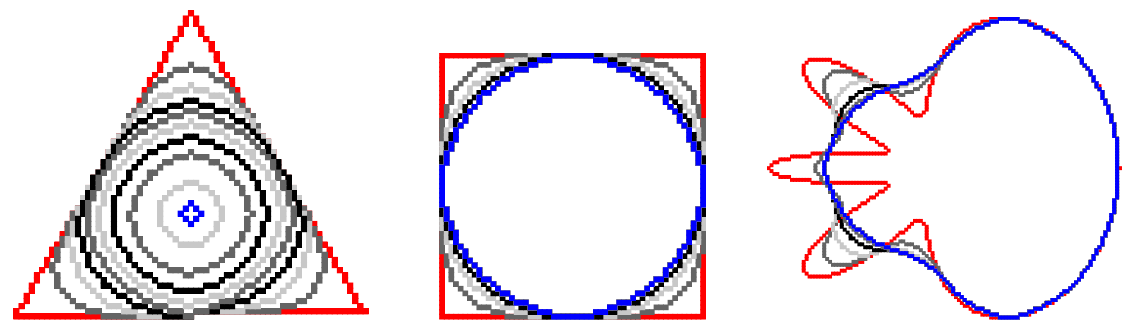

(b) $r=5, h=0.5$

$h=0.5 r=5$

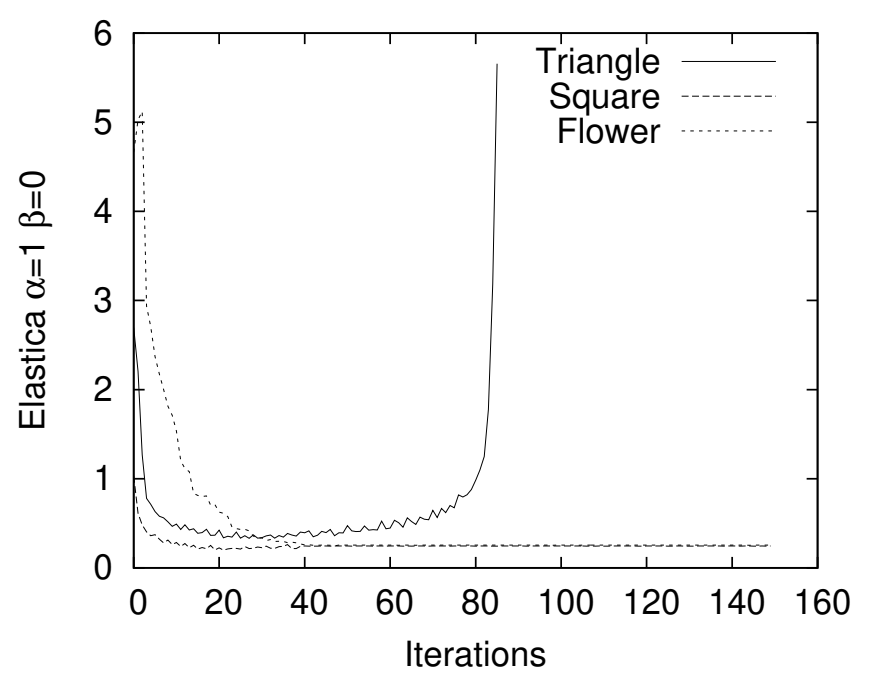

(c) Elastica evaluation

Fig. 6: The choice of radius impacts the flow. In the figures, the flow ceases to evolve for all shapes when $r=3$ (a). In figure (b), for $r=5$, the triangle evolves to a single point, while the others stop in an intermediate shape, as in (a). In figure (c), we observe that for a given choice of radius, the elastica may increase after a certain number of iterations. 


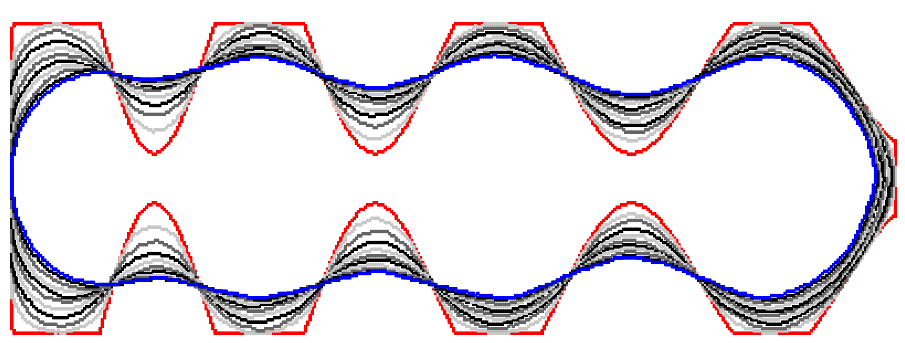

(a)

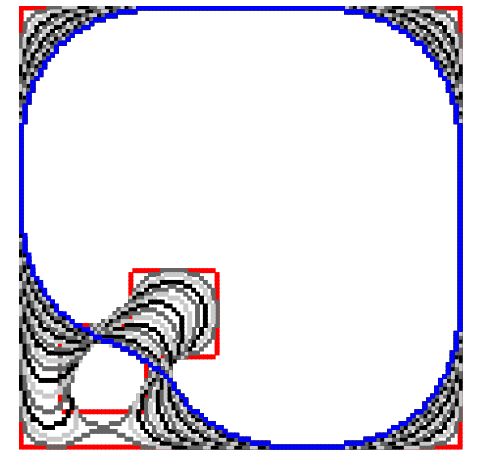

(b)

Fig. 7: High curvature regions evolves faster than lower ones (a). The flow can handle topological changes (b).

(probe). The first is an approximation method that is guaranteed to not increase the energy, but loses the property of partial optimality. The second is an exact method which is reported to label more variables than QPBO.

The percentage of unlabeled pixels by QPBOP for $E_{1}$ is quite high, but the percentage decreases to zero as we set $m$ equal to $r$. Therefore, we are more confident in taking the solution for values of $m$ close to $r$. However, the way it varies across $m$ values differs from shape to shape, as is illustrated in figure 9 . We also noticed that, for $m=r$, all the pixels were labeled, which may indicate that $E_{r}$ is an an easy instance of the general non-submodular energy $E_{m}$, but this remains to be proved. The number of pairwise terms in $E_{r}$ is roughly half of those in $E_{1}$ (see figure 8).

We have used QPBOI to solve $E_{m}$. Naturally, in the case where all pixels are labeled by QPBOP, QPBOI returns the same labeling as QPBOP.

\section{Application to image segmentation}

We present an application of our digital curve evolution algorithm to supervised image segmentation. The DCE acts as a contour correction method. Here we use a data fidelity term in order to characterize the object of interest. 


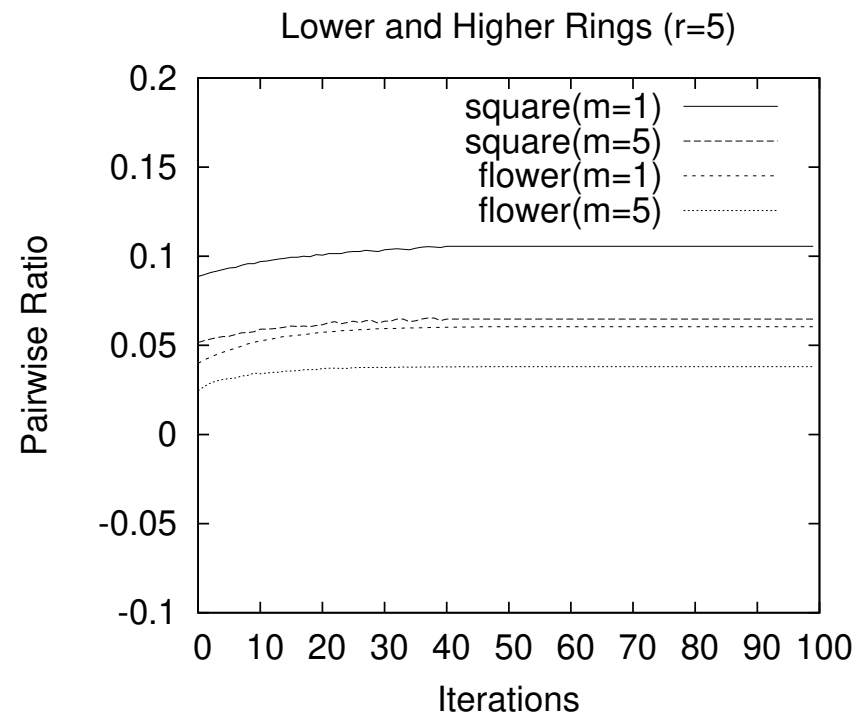

Fig. 8: We plot the ratio of pairwise terms among all $\left(\begin{array}{c}\left|X^{(i)}\right| \\ 2\end{array}\right)$ combinations. The highest ring has rougly half the number of pairwise terms than the lowest ring.

Given foreground and background seeds selected by the user, we derive mixed gaussian distributions of color intensities $G_{f}$ and $G_{b}$, and we define the data fidelity term as the cross-entropy, i.e.

$$
g(y)=-(1-y) \log G_{f}(I(y))-y \log G_{b}(I(y))
$$

We use the DCE algorithm to regularize an initial contour outputed by some segmentation algorithm or delineated by the user. In this application, the similarity term of the DCE is set to the data fidelity term (8). 


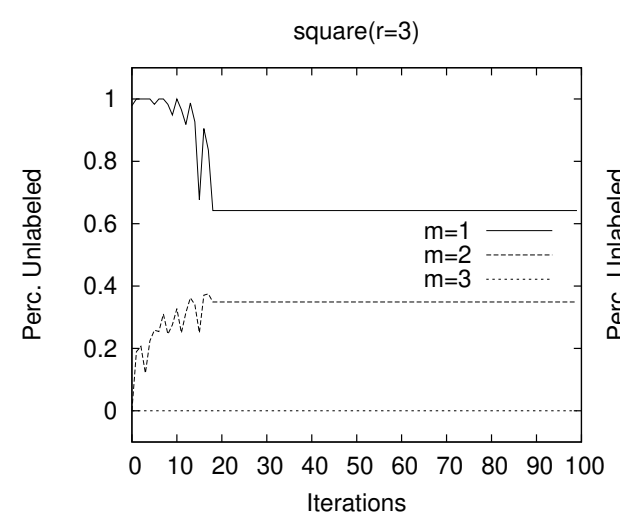

(a)

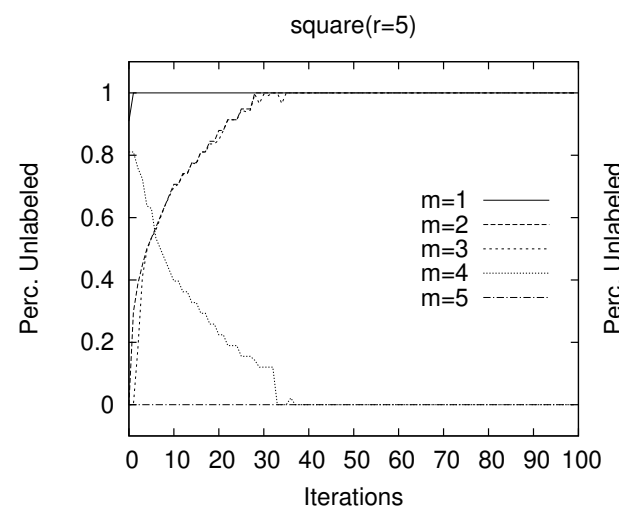

(b)

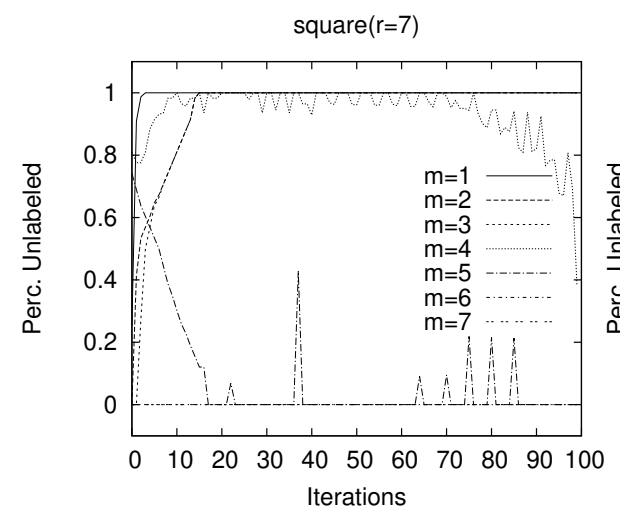

(c)

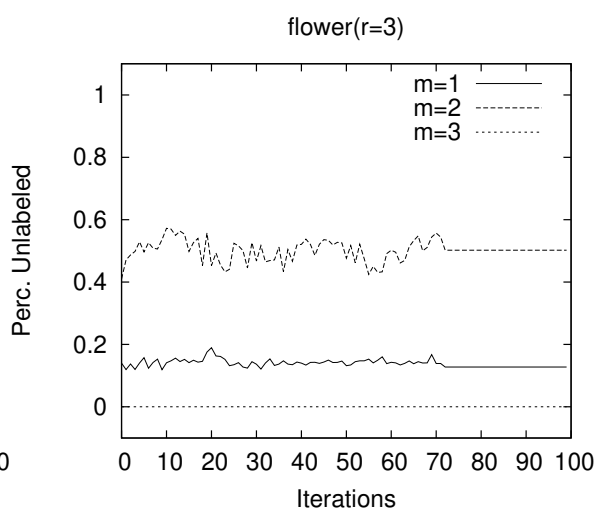

(d)

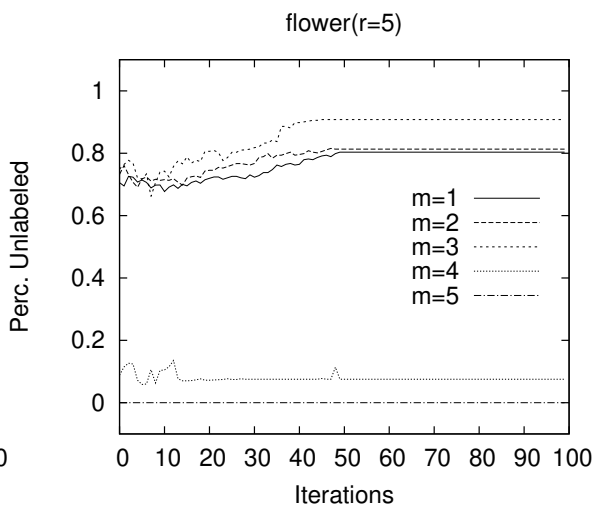

(e)

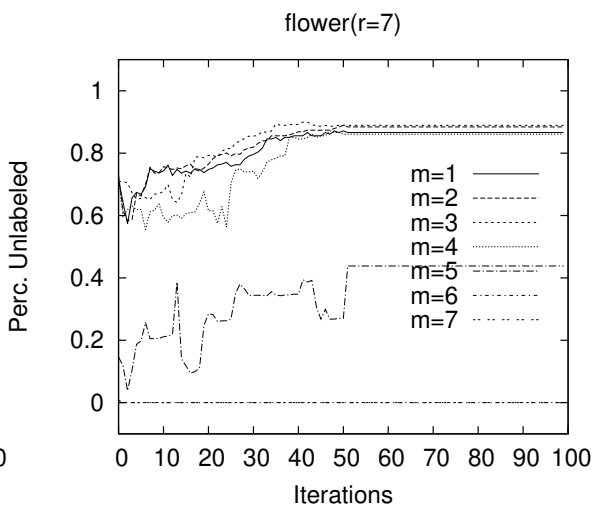

(f)

Fig. 9: For each plot, we first produce shapes $\left\{S^{(i)}\right\}$ executing DCE with $m=r$. Then, for each shape in $\left\{S^{(i)}\right\}$, we execute one iteration of DCE for different values of $m$ and we count the unlabeled pixels. The number of unlabeled pixels by QPBOP remains high for lower values of $m$, and goes to zero when $m=r$. We observe the same behaviour for varying radius values. 


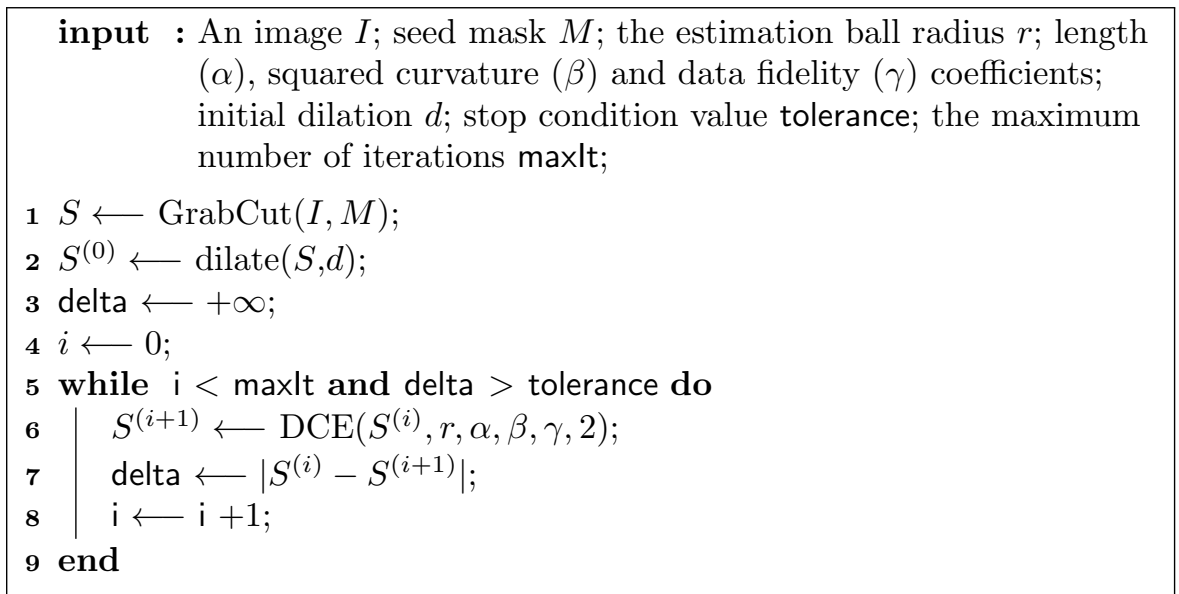

Algorithm 3: Contour correction algorithm.

The algorithm can be initialized by a collection of compact sets, or with the result of a third-party segmentation algorithm, as GrabCut [34]. We include an additional parameter $d$ that dilates the initial sets using a square of side one before executing the flow.

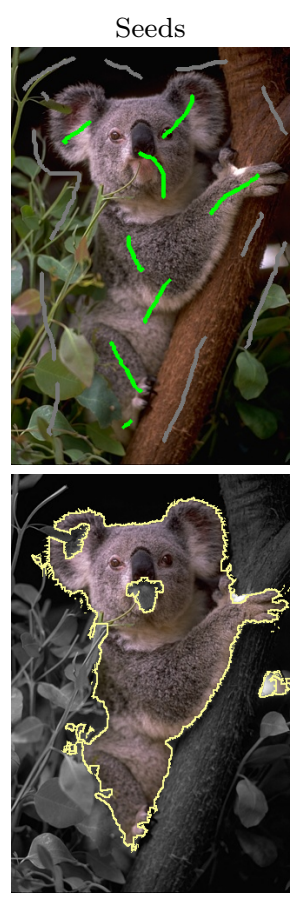

GrabCut
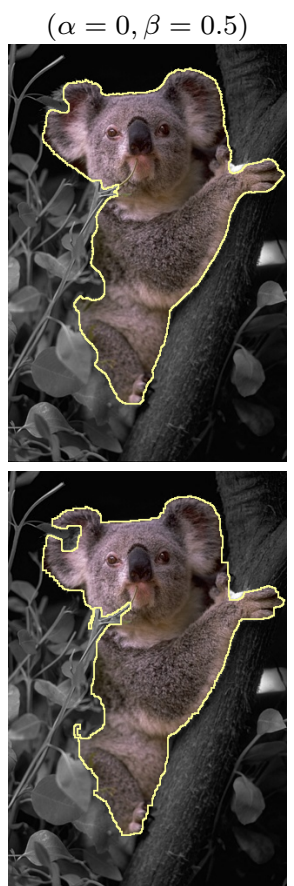

$(\alpha=0.5, \beta=0)$
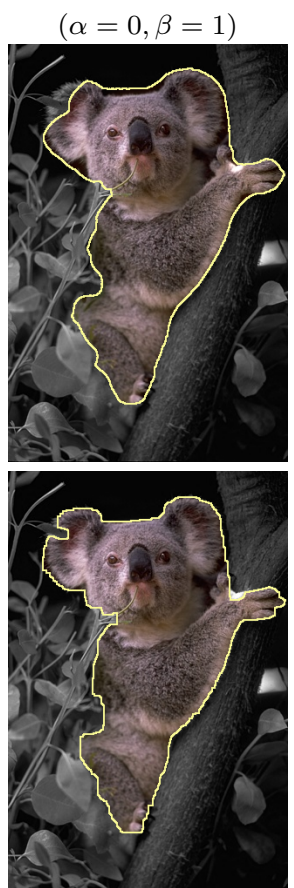

$(\alpha=1, \beta=0)$

Fig. 10: Comparison of squared curvature regularization (first row) and length regularization (second row). 


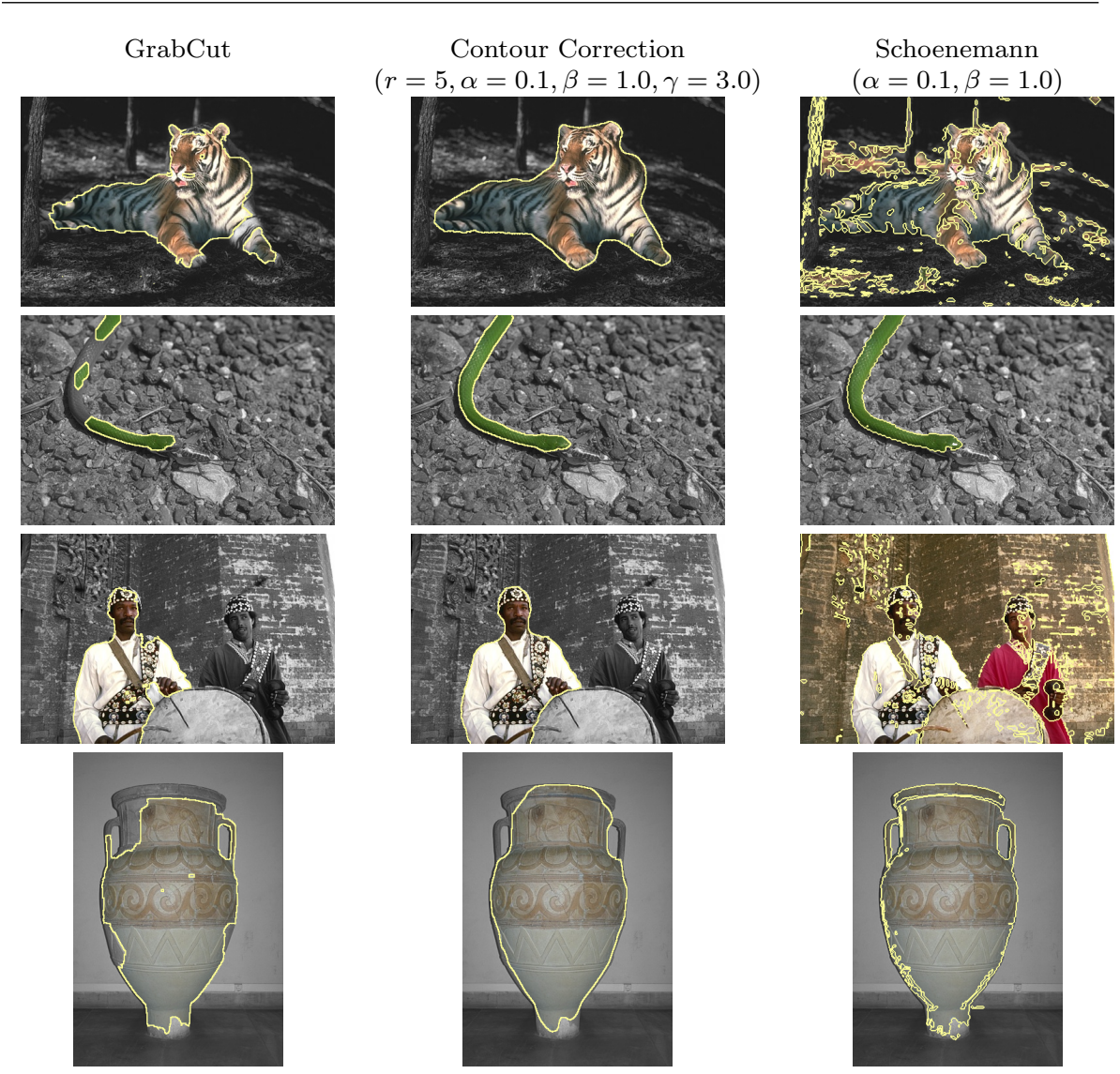

Fig. 11: The proposed method regularizes GrabCut contours and returns meaningful results. We can observe the completion feature of curvature in the second row, and we don't suffer from oversegmentation issues as Schoenemann's method. However, our flow may stop in a local optima as in the fourth row, while Schoenemann's is able to extrapolate such solutions.

We evaluate our method using the BSD300 database [29]. All images contains the same number of pixels, the resolution being $321 \times 481$ in portrait mode. We compare the results of our method with segmentations given by GrabCut and Schoenemanns's method [38]. We report an average of $3 s$ per flow iteration, and an average of 30 iterations per image. While GrabCut executes in less than one second, Schoenemann's method may take several hours to complete.

In figure 10 we can observe the results of a curvature regularization in comparison with a pure length regularization. The curvature can fill gaps and is smoother than the one produced by length only, resulting in more pleasant segmentations. In figures 11 and 12 we list several results of our method and we compare them with segmentation produced by GrabCut and Schoenemann's method. Our method is successful in producing curvature regularized segmen- 
GrabCut
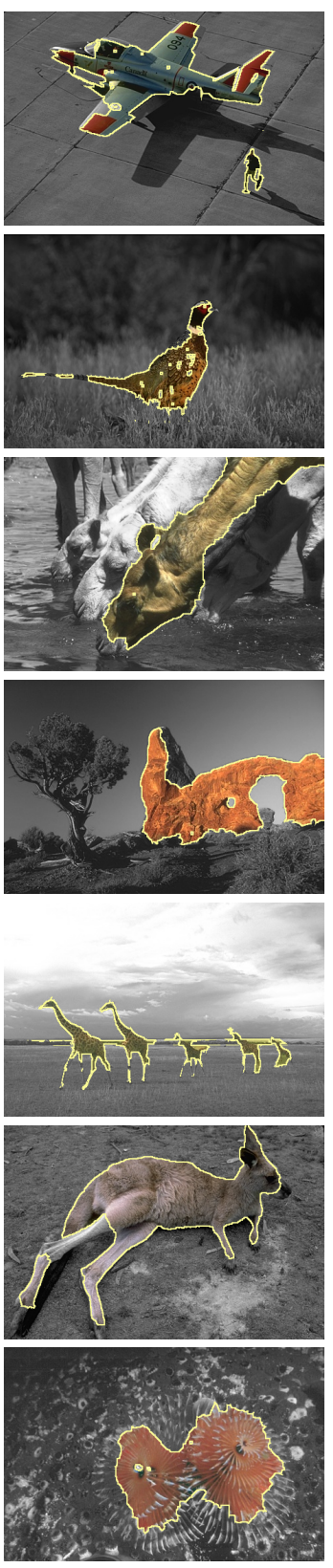

Contour Correction

$(r=5, \alpha=0.1, \beta=1.0, \gamma=3.0)$
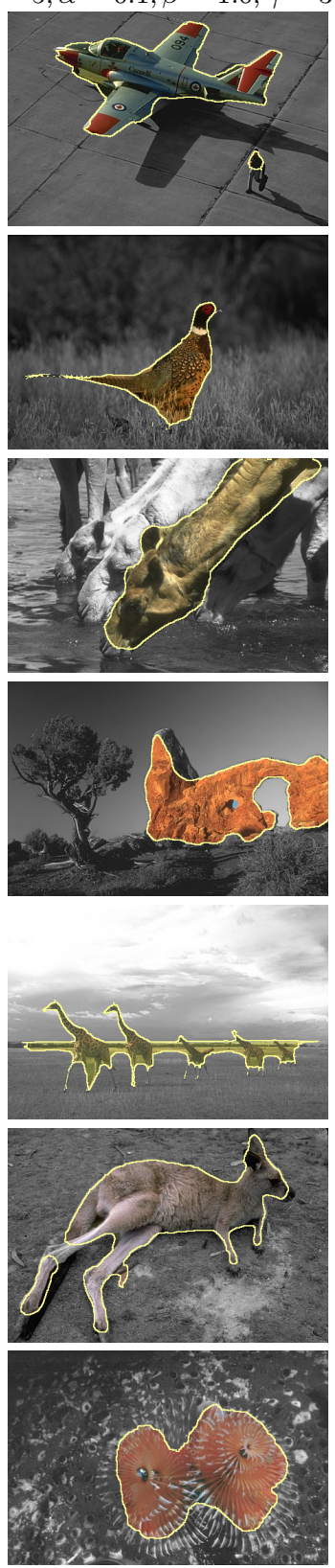

Fig. 12
Schoenemann

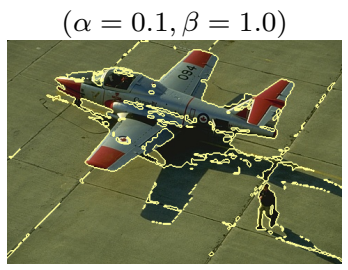

Fen

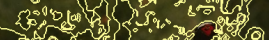

Q.
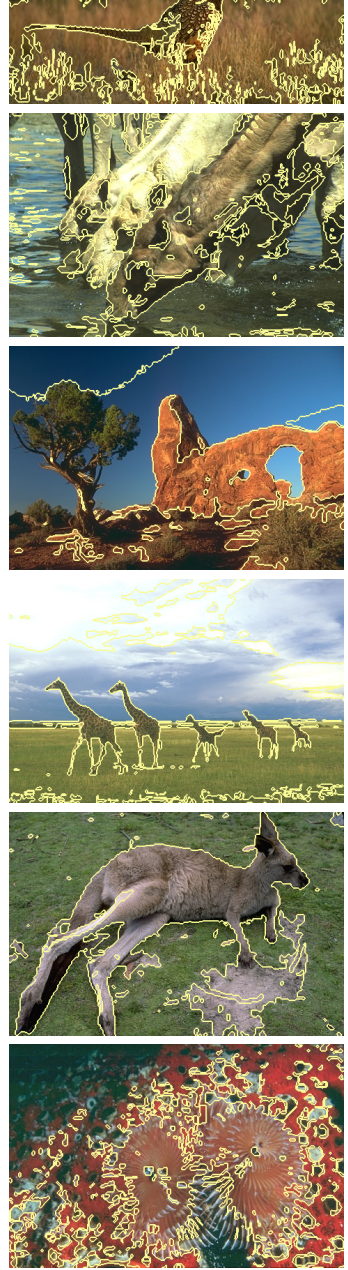
tations and demonstrates the completion property of curvature. Moreover, it does not suffer from over segmentation, it is much faster than Schoenemann's, and in several opportunities produces better segmentations than GrabCut.

\section{Conclusion}

We have studied in depth several digital curve evolution models based on a digital version of the Elastica energy and we have presented an application to image segmentation. The processes we have described are completely digital, and do not suffer from issues that typically arise in models that passes through a discretization stage, such as rounding. Moreover, the model can handle changes in topology and its results are competitive with similar approaches while achieving reasonable running times.

Future developments of this work will include extending the DCE algorithm to $3 \mathrm{~d}$, as the integral invariant estimator is available in higher dimensions. As a result, a denoising application may be derived by lifting the flow to $3 \mathrm{~d}$ with a data fidelity defined by image intensities or by distance to initial position.

\section{References}

1. Antunes, D., Lachaud, J.O., Talbot, H.: Digital curvature evolution model for image segmentation. In: Couprie, M., Cousty, J., Kenmochi, Y., Mustafa, N. (eds.) Discrete Geometry for Computer Imagery. pp. 15-26. Springer International Publishing, Cham (2019)

2. Appleton, B., Talbot, H.: Globally optimal geodesic active contours. Journal of Mathematical Imaging and Vision 23(1), 67-86 (Jul 2005)

3. Ballester, C., Bertalmio, M., Caselles, V., Sapiro, G., Verdera, J.: Filling-in by joint interpolation of vector fields and gray levels. IEEE Transactions on Image Processing 10(8), 1200-1211 (Aug 2001). https://doi.org/10.1109/83.935036

4. Bobenko, A.I., Schröder, P.: Discrete willmore flow (2005)

5. Boykov, Y.Y., Jolly, M.P.: Interactive graph cuts for optimal boundary amp; region segmentation of objects in n-d images. In: Proceedings Eighth IEEE International Conference on Computer Vision. ICCV 2001. vol. 1, pp. 105-112 vol.1 (2001)

6. Bredies, K., Pock, T., Wirth, B.: A convex, lower semicontinuous approximation of euler's elastica energy. SIAM Journal on Mathematical Analysis 47, 566-613 (01 2015). https://doi.org/10.1137/130939493

7. Bretin, E., Masnou, S., Oudet, E.: Phase-field approximations of the willmore functional and flow. Numerische Mathematik 131(1), 115-171 (2015)

8. Caselles, V., Kimmel, R., Sapiro, G.: Geodesic active contours. International Journal of Computer Vision 22(1), 61-79 (Feb 1997)

9. Chan, T.F., Kang, S.H., Kang, Shen, J.: Euler's elastica and curvature based inpaintings. SIAM J. Appl. Math 63, 564-592 (2002)

10. Coeurjolly, D., Klette, R.: A comparative evaluation of length estimators of digital curves. IEEE Transactions on Pattern Analysis and Machine Intelligence 26(2), 252258 (Feb 2004)

11. Coeurjolly, D., Lachaud, J.O., Levallois, J.: Integral based curvature estimators in digital geometry. In: Gonzalez-Diaz, R., Jimenez, M.J., Medrano, B. (eds.) Discrete Geometry for Computer Imagery. pp. 215-227. Springer Berlin Heidelberg, Berlin, Heidelberg (2013)

12. Coeurjolly, D., Lachaud, J.O., Roussillon, T.: Multigrid Convergence of Discrete Geometric Estimators, pp. 395-424. Springer Netherlands, Dordrecht (2012) 
13. Deng, L.J., Glowinski, R., Tai, X.C.: A new operator splitting method for the euler elastica model for image smoothing. SIAM Journal on Imaging Sciences 12(2), 11901230 (2019)

14. Droske, M., Rumpf, M.: A level set formulation for willmore flow. Interfaces and free boundaries 6(3), 361-378 (2004)

15. El-Zehiry, N.Y., Grady, L.: Fast global optimization of curvature. In: 2010 IEEE Computer Society Conference on Computer Vision and Pattern Recognition. pp. 3257-3264 (June 2010)

16. El-Zehiry, N.Y., Grady, L.: Contrast driven Elastica for image segmentation. IEEE Transactions on Image Processing 25(6), 2508-2518 (2016)

17. Esedoglu, S., Ruuth, S., Tsai, R.: Threshold dynamics for shape reconstruction and disocclusion. In: IEEE International Conference on Image Processing 2005. vol. 2, pp. II-502. IEEE (2005)

18. Esedoglu, S., Ruuth, S.J., Tsai, R.: Threshold dynamics for high order geometric motions. Interfaces and Free Boundaries 10(3), 263-282 (2008)

19. Goldluecke, B., Cremers, D.: Introducing total curvature for image processing. In: 2011 International Conference on Computer Vision. pp. 1267-1274 (Nov 2011). https://doi.org/10.1109/ICCV.2011.6126378

20. Kass, M., Witkin, A., Terzopoulos, D.: Snakes: Active contour models. International journal of computer vision 1(4), 321-331 (1988)

21. Klette, R., Žunić, J.: Multigrid convergence of calculated features in image analysis. Journal of Mathematical Imaging and Vision 13(3), 173-191 (2000)

22. Kolmogorov, V., Zabin, R.: What energy functions can be minimized via graph cuts? IEEE Transactions on Pattern Analysis and Machine Intelligence 26(2), 147-159 (Feb 2004)

23. Lachaud, J.O.: Non-Euclidean spaces and image analysis : Riemannian and discrete deformable models, discrete topology and geometry. Ph.D. thesis, Université Sciences et Technologies - Bordeaux I (Dec 2006), https://tel.archives-ouvertes.fr/tel-00396332

24. Lachaud, J.O., Vialard, A., de Vieilleville, F.: Fast, accurate and convergent tangent estimation on digital contours. Image Vision Comput. 25(10), 1572-1587 (Oct 2007)

25. Lim, P.H., Bagci, U., Bai, L.: Introducing willmore flow into level set segmentation of spinal vertebrae. IEEE Transactions on Biomedical Engineering 60(1), 115-122 (2012)

26. Malladi, R., Sethian, J.A.: Image processing via level set curvature flow. proceedings of the National Academy of sciences 92(15), 7046-7050 (1995)

27. Malladi, R., Sethian, J.A., Vemuri, B.C.: Shape modeling with front propagation: A level set approach. IEEE T. Patt. Anal. Mach. Intell. 17(2) (1995)

28. Manay, S., Hong, B.W., Yezzi, A.J., Soatto, S.: Integral invariant signatures. In: Pajdla, T., Matas, J. (eds.) Computer Vision - ECCV 2004. pp. 87-99. Springer Berlin Heidelberg, Berlin, Heidelberg (2004)

29. Martin, D., Fowlkes, C., Tal, D., Malik, J.: A database of human segmented natural images and its application to evaluating segmentation algorithms and measuring ecological statistics. In: Proc. 8th Int'l Conf. Computer Vision. vol. 2, pp. 416-423 (July 2001)

30. Masnou, S., Morel, J.M.: Level lines based disocclusion. In: Proceedings 1998 International Conference on Image Processing. ICIP98 (Cat. No.98CB36269). pp. 259-263 vol.3 (Oct 1998)

31. Mumford, D.: Elastica and computer vision. In: Algebraic geometry and its applications, pp. 491-506. Springer (1994)

32. Nieuwenhuis, C., Toeppe, E., Gorelick, L., Veksler, O., Boykov, Y.: Efficient squared curvature. In: 2014 IEEE Conference on Computer Vision and Pattern Recognition. pp. 4098-4105 (June 2014)

33. Pottmann, H., Wallner, J., Huang, Q.X., Yang, Y.L.: Integral invariants for robust geometry processing. Computer Aided Geometric Design 26(1), 37 - 60 (2009)

34. Rother, C., Kolmogorov, V., Blake, A.: "grabcut": Interactive foreground extraction using iterated graph cuts. ACM Trans. Graph. 23(3), 309-314 (Aug 2004)

35. Rother, C., Kolmogorov, V., Lempitsky, V.S., Szummer, M.: Optimizing binary mrfs via extended roof duality. 2007 IEEE Conference on Computer Vision and Pattern Recognition pp. 1-8 (2007) 
36. Roussillon, T., Lachaud, J.O.: Accurate curvature estimation along digital contours with maximal digital circular arcs. In: Aggarwal, J.K., Barneva, R.P., Brimkov, V.E. Koroutchev, K.N., Korutcheva, E.R. (eds.) Combinatorial Image Analysis. pp. 43-55. Springer Berlin Heidelberg, Berlin, Heidelberg (2011)

37. Schindele, A., Massopust, P., Forster, B.: Multigrid convergence for the MDCA curvature estimator. J. Math. Imaging Vis. 57(3), 423-438 (Mar 2017)

38. Schoenemann, T., Kahl, F., Cremers, D.: Curvature regularity for region-based image segmentation and inpainting: A linear programming relaxation. In: 2009 IEEE 12th International Conference on Computer Vision. pp. 17-23 (Sept 2009)

39. Schoenemann, T., Masnou, S., Cremers, D.: The elastic ratio: Introducing curvature into ratio-based image segmentation. IEEE Transactions on Image Processing 20(9), 2565-2581 (2011)

40. Strandmark, P., Kahl, F.: Curvature regularization for curves and surfaces in a global optimization framework. In: Boykov, Y., Kahl, F., Lempitsky, V., Schmidt, F.R. (eds.) Energy Minimization Methods in Computer Vision and Pattern Recognition. pp. 205218. Springer Berlin Heidelberg, Berlin, Heidelberg (2011)

41. Tai, X.C., Hahn, J., Chung, G.J.: A fast algorithm for euler's elastica model using augmented lagrangian method. SIAM J. Img. Sci. 4(1), 313-344 (Mar 2011)

42. Zhu, W., Tai, X.C., Chan, T.: Image segmentation using euler's elastica as the regularization. Journal of scientific computing 57(2), 414-438 (2013) 